\title{
Rapid High Resolution Combined AFM and Raman Microanalysis
}

Thomas J Tague Jr., Ph.D. and David L. Sampson, Ph.D.

Bruker Optics, Inc., 19 Fortune Drive, Billerica, MA 01821

Bruker Nano, Inc., 5465 East Cheryl Parkway, Madison, WI 53711

Atomic force microscopy (AFM) has been commonly used for many years in characterizing the surface of materials of interest. The fabrication of semiconductors and nanostructures has been an area of significant application of AFM, where imperfections due to stress and strain area readily detected due to eruptions at the surface. For example, when $\mathrm{GaN}$ is grown on Sapphire, differences in the coefficients of thermal expansion cause fractures in the GaN film, precluding the fabrication of $\mathrm{GaN}$ thins films. The fractures are readily observed by AFM. Figure 1 shows an AFM image of single walled nanotubes grown on silicon. The field of view (FOV) for the AFM image is $3 \times 3$ microns and the diameter of the nanotubes is determined to be about $100 \mathrm{~nm}$. The sample did not have to be moved in switching between collecting the AFM image and the subsequent Raman analysis. The AFM measurement was accomplished by utilizing a novel AFM scanner that attaches to the nosepiece of the Raman microscope. The AFM utilizes an interferometric cantilever deflection detection system instead of the more prevalent laser beam bounce deflection system. This approach has technical as well as operational benefits. Primarily, the cantilever position is measured directly, calculations and approximations are unnecessary to determine precise cantilever deflection or oscillation amplitude leading to more reliable force control. The Raman spectrum was collected at $3 \mathrm{~cm}^{-1}$ spectral resolution with $532 \mathrm{~nm}$ excitation.

In the late 1830's, Daguerro found that if a copper plate coated with silver iodide is exposed to light, then fumed with mercury vapor and fixed using a solution of common salt, a permanent image is formed. This was the first successful form of photography. Surviving daguerrotype plates are therefore of significant historical value. Figure 2 shows a daguerrotype plate that unfortunately suffered significant degradation during a 10-month exhibition. AFM and Raman images were collected to elucidate the origin and mechanics of the degradation. Raman proved to be very sensitive due to the fact that the silver Ag surface acts as surface-enhanced Raman scattering (SERS) substrate. The band at $243 \mathrm{~cm}^{-1}$, assigned to the $\mathrm{Ag}-\mathrm{Cl}$ stretching mode, has been observed in the areas where a white surface layer or white spots are present. The presence of $\mathrm{Ag}-\mathrm{Cl}$ bonds on daguerreotype surfaces has profound implications for their exhibition and preservation due to the photosensitivity of silver chloride in the UV range. 

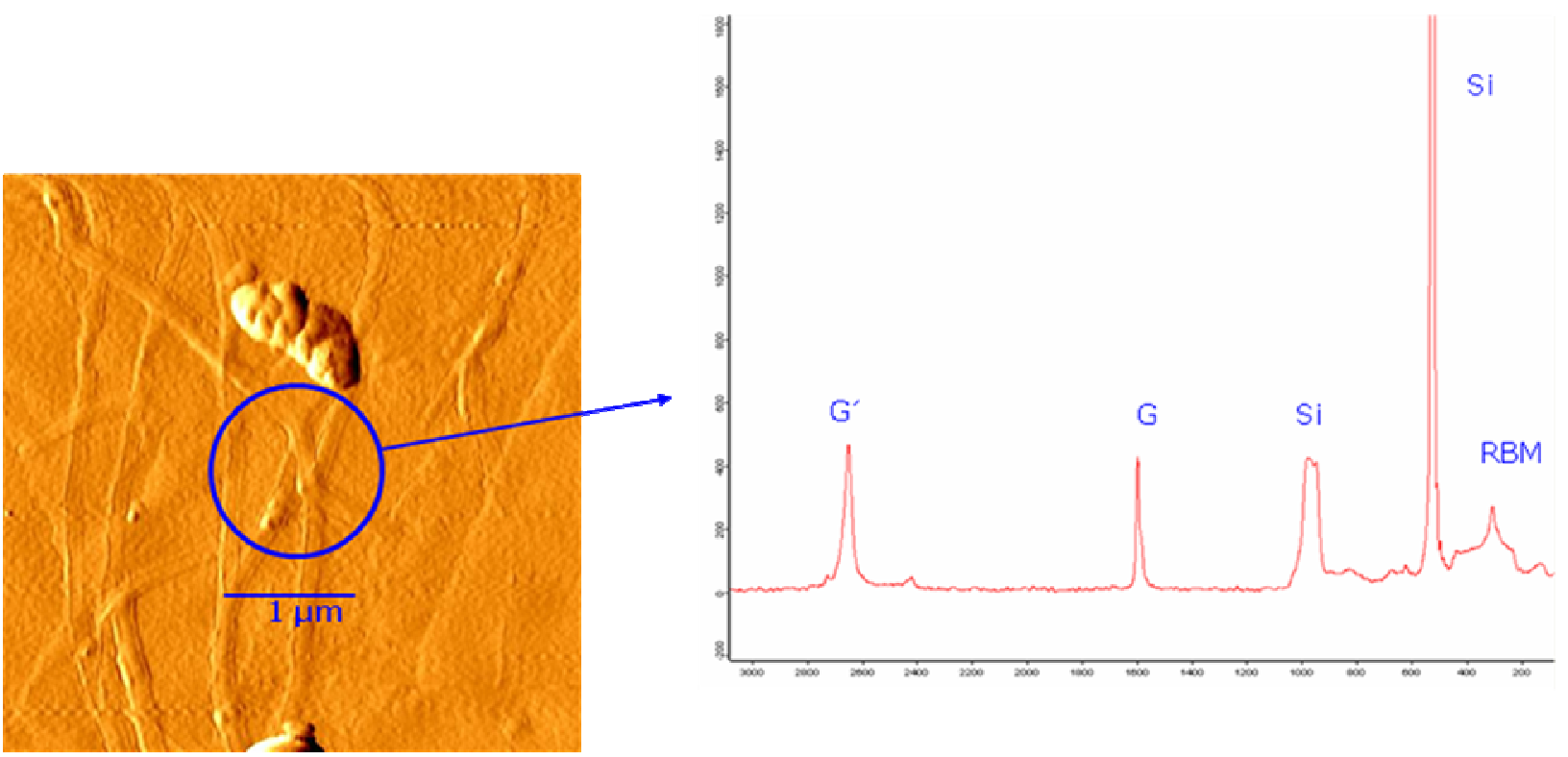

Figure 1 - An AFM image of nanotubes grown on silicon is shown (left), as well as the resulting Raman spectrum (right).
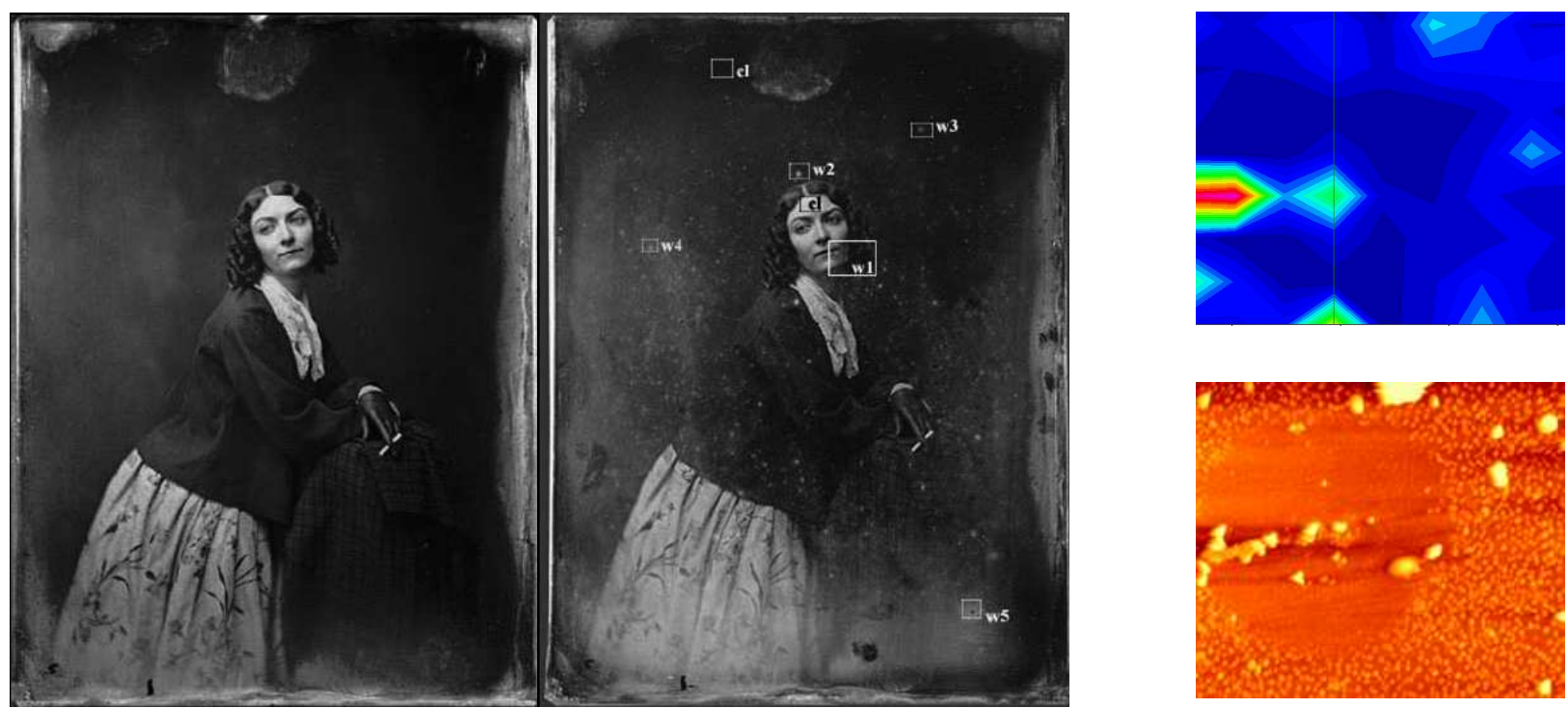

Figure 2 - The above images shows a daguerrotype plate before (left) and after (center) a 10 month-long exhibition ${ }^{\mathrm{i}}$. The AFM (lower right) and Raman (upper right) analysis were performed in the area shown by the w4. Shown with permission from The Metropolitan Museum of Art.

' - S. A. Centeno, T. Miller, N. Kennedy, and M. Wypyski J. Raman Spectrosc. 2008; 39: 914-921 